\title{
ON THE RECOMBINATION CURRENTS EFFECT OF HETEROSTRUCTURE-EMITTER BIPOLAR TRANSISTORS (HEBTs)
}

\author{
JUNG-HUI TSAI', LIH-WEN LAIH ${ }^{1}$, HUI-JUNG SHIH ${ }^{1}$, WEN-CHAU LIU ${ }^{1}$ and \\ HAO-HSIUNG LIN ${ }^{2}$
}

'Department of Electrical Engineering, National Cheng-Kung University, 1 University Road, Tainan, Taiwan, Republic of China and 'Department of Electrical Engineering, National Taiwan University, Taipei, Taiwan, Republic of China

(Received 12 June 1996)

\begin{abstract}
In this paper, we will demonstrate the effect of recombination current on the electrical properties of heterostructure-emitter bipolar transistors (HEBTs). For comparison, an AlGaAs/GaAs and an AlInAs/GaInAs HEBT are fabricated with the same layer structure. The theoretical analysis shows that the neutral-emitter recombination current in the neutral emitter regime is a significant factor for determining transistor characteristics. For the AlGaAs/GaAs HEBT, the hole diffusion length is larger than the emitter thickness, so that most of holes can be reflected back at the confinement layer due to the hole recombination current being low in the neuter-emitter region. Thus, the high emitter injection efficiency and current gain can be achieved simultaneously. On the other hand, for the AlInAs/GaInAs HEBT, the increase of recombination current at neutral emitter regime and the existence of potential spike could reduce the emitter injection efficiency at large $V_{\mathrm{BE}}$ voltage. Hence, the non-1KT component of collector current is enhanced and the characteristics of transistor are degraded. However, a lower offset voltage of $40 \mathrm{mV}$ is obtained attributed to the low base surface recombination current for the AlInAs/GaInAs HEBT. All of these experimental results are consistent with the theoretical analysis. Copyright (C) 1996 Elsevier Science Ltd
\end{abstract}

\section{INTRODUCTION}

The heterojunction bipolar transistors (HBTs) have attracted considerable attention for digital and microwave power applications due to their high speed and high current handling capabilities[1-3]. The basic concept of HBT is employing a wide-gap emitter to introduce an energy barrier that limits the injection of minority carrier from base region. Therefore, the benefits of HBT are not only the ability to achieve high emitter injection efficiency and hence high current gain, but also the freedom to change doping levels in emitter and base without significant constraints of injection efficiency consideration. However, there are some drawbacks including the difficulty in precise alignment between the composition junction and doping junction attributed to the out-diffusion of p-type dopants, and the larger emitter-collector $(E-C)$ offset voltage resulting in unnecessary power consumption. In order to overcome the above problems, a heterostructureemitter bipolar transistor (HEBT) has been proposed and successfully fabricated by Luo et al.[4] and Liu and Lour[5]. In the HEBT, there is a homojunction emitter with a heterojunction confinement layer on top of it. The heterostructure is used as minority carrier (hole) confinement barrier and is physically separated from the emitter-base (E-B) $p-n$ junction.
The homojunction controls the electron injection. Moreover, an emitter layer is used to avoid the p-dopant out-diffusion as well as the presence of potential spike.

Recently, it has been shown that $\mathrm{AlGaAs} / \mathrm{GaAs}$ and InGaP/GaAs HEBTs are attractive for high current gain performance and high frequency applications [5-7]. Chen et al.[6] have demonstrated the minimum emitter thickness of $\mathrm{AlGaAs} / \mathrm{GaAs}$ HEBTs to eliminate the potential spike and the appropriate emitter edge-thinning thickness to achieve the high current gain performances. The InGaP/GaAs based HEBT was first fabricated by Yang et al.[7]. They demonstrated the significant lower recombination current and higher current gain with an InGaP passivation layer. It is known that the emitter layer thickness is an important factor for HEBT characteristics. However, owing to the insertion of n-emitter layer, the charge storage effect is enhanced and the emitter injection efficiency and current gain may be degraded.

Previously, some theoretical analysis of HBT devices have been reported[8-10]. However, the recombination current effect on the HEBTs has still not been investigated clearly. In this paper, we will report the influences of recombination current on the HEBT characteristics. Two different material-based HEBTs, i.e. AlGaAs/GaAs and AlInAs/GaInAs 
HEBTs, with the same layer structure are fabricated and compared. In the next section, the device fabrication process is presented. In Sections 3 and 4, the theoretical analysis and experimental results are demonstrated and discussed, respectively. The effect of base-current components on the studied devices is also reported. Finally, a conclusion is given.

\section{DEVICES FABRICATION AND EXPERIMENTS}

The studied structures were grown by molecular beam epitaxy (MBE). The n-type and p-type dopant used here are $\mathrm{Si}$ and $\mathrm{Be}$, respectively. The AlGaAs/GaAs HEBT, grown on a (100)-oriented $\mathrm{n}^{+}-\mathrm{GaAs}$ substrate, consisted of a $0.2 \mu \mathrm{m}$ $\mathrm{n}^{+}=1 \times 10^{18} \mathrm{~cm}^{-3}$ GaAs buffer layer, a $0.5 \mu \mathrm{m}$ $\mathrm{n}^{-}=5 \times 10^{16} \mathrm{~cm}^{-3} \quad$ GaAs collector, a $0.2 \mu \mathrm{m}$ $\mathbf{p}^{+}=5 \times 10^{18} \mathrm{~cm}^{-3} \quad$ GaAs base, a $500 \AA$ $\mathrm{n}=5 \times 10^{17} \mathrm{~cm}^{-3} \quad$ GaAs emitter, a $0.1 \mu \mathrm{m}$ $\mathrm{n}=5 \times 10^{17} \mathrm{~cm}^{-3}$ AlGaAs confinement layer and a $0.3 \mu \mathrm{m} \mathrm{n}^{+}=3 \times 10^{18} \mathrm{~cm}^{-3}$ GaAs cap layer. The $\mathrm{Al}_{0.48} \mathrm{In}_{0.52} \mathrm{As} / \mathrm{Ga}_{0.47} \mathrm{In}_{0.53} \mathrm{As} \mathrm{HEBT}$ was grown on a (100)-oriented $n^{+}$-InP substrate. The layer structure of AlInAs/GaInAs HEBT is similar to AlGaAs/ GaAs HEBT including a $0.2 \mu \mathrm{m} \mathrm{n} n^{+}=1 \times 10^{18} \mathrm{~cm}^{-3}$ GaInAs buffer layer, a $0.5 \mu \mathrm{m} \mathrm{n} \mathrm{n}^{-}=5 \times 10^{16} \mathrm{~cm}^{-3}$ GaInAs collector, a $0.2 \mu \mathrm{m} \quad \mathrm{p}^{+}=5 \times 10^{18} \mathrm{~cm}^{-3}$ GaInAs base, a $500 \AA \mathrm{n}=5 \times 10^{17} \mathrm{~cm}^{-3}$ GaInAs emitter, a $0.1 \mu \mathrm{m} \mathrm{n}=5 \times 10^{17} \mathrm{~cm}^{-3}$ AlInAs confinement layer and a $0.3 \mu \mathrm{m} \mathrm{n}^{+}=3 \times 10^{18} \mathrm{~cm}^{-3}$ GaInAs cap layer. After MBE growth, the wet-etching and photolithographic process were used to define the emitter and the base regions. Ohmic contacts were performed by alloying evaporated AuGe and AuZn metals for the n-type emitter, collector and p-type base, respectively. The emitter areas are $5.1 \times 10^{-5} \mathrm{~cm}^{-2}$ and $4.9 \times 10^{-5} \mathrm{~cm}^{-2}$ for AlGaAs/ GaAs and AlInAs/GaInAs HEBTs, respectively.

\section{THEORETICAL ANALYSIS}

For the HEBT structure, the confinement layer is used as a confinement barrier for holes and separated from the $\mathrm{E}-\mathrm{B}$ junction. If the emitter layer is too small, the potential spike could exist and the collector-emitter offset voltage increase. Thus, a minimum emitter layer thickness must be found to eliminate the undesired potential spike. By solving the Poisson's equation, the minimum emitter layer thickness can be obtained as[6]

$$
a=\sqrt{\frac{2 \varepsilon \Delta E_{\mathrm{c}}}{q^{2} N_{\mathrm{D}}}}\left(\frac{N_{\mathrm{A}}}{N_{\mathrm{A}}+N_{\mathrm{D}}}\right)
$$

where $\Delta E_{\mathrm{c}}, N_{\mathrm{D}}$ and $N_{\mathrm{A}}$ are the conduction band discontinuity between confinement and emitter layer, emitter and base layer concentration, respectively.

From calculation, the values of $a$ are 260 and $390 \AA$ for the AlGaAs/GaAs and AlInAs/GaInAs HEBT, respectively. Hence, a $500 \AA$ emitter layer thickness is sufficient to eliminate the potential spike at small $V_{\mathrm{BE}}$ voltage and reduce the offset voltage for the studied

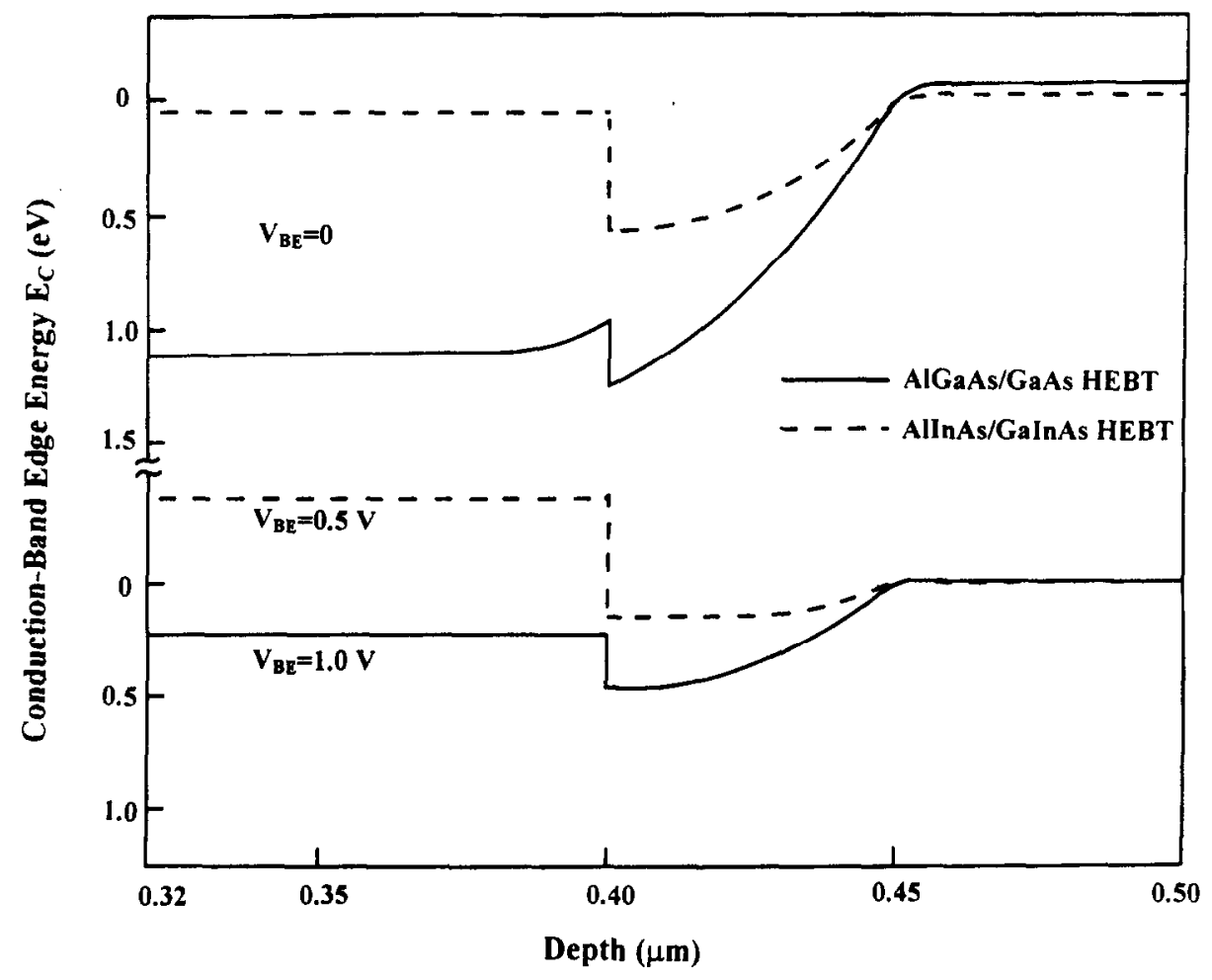

Fig. 1. The calculated conduction band diagram for the studied devices. 


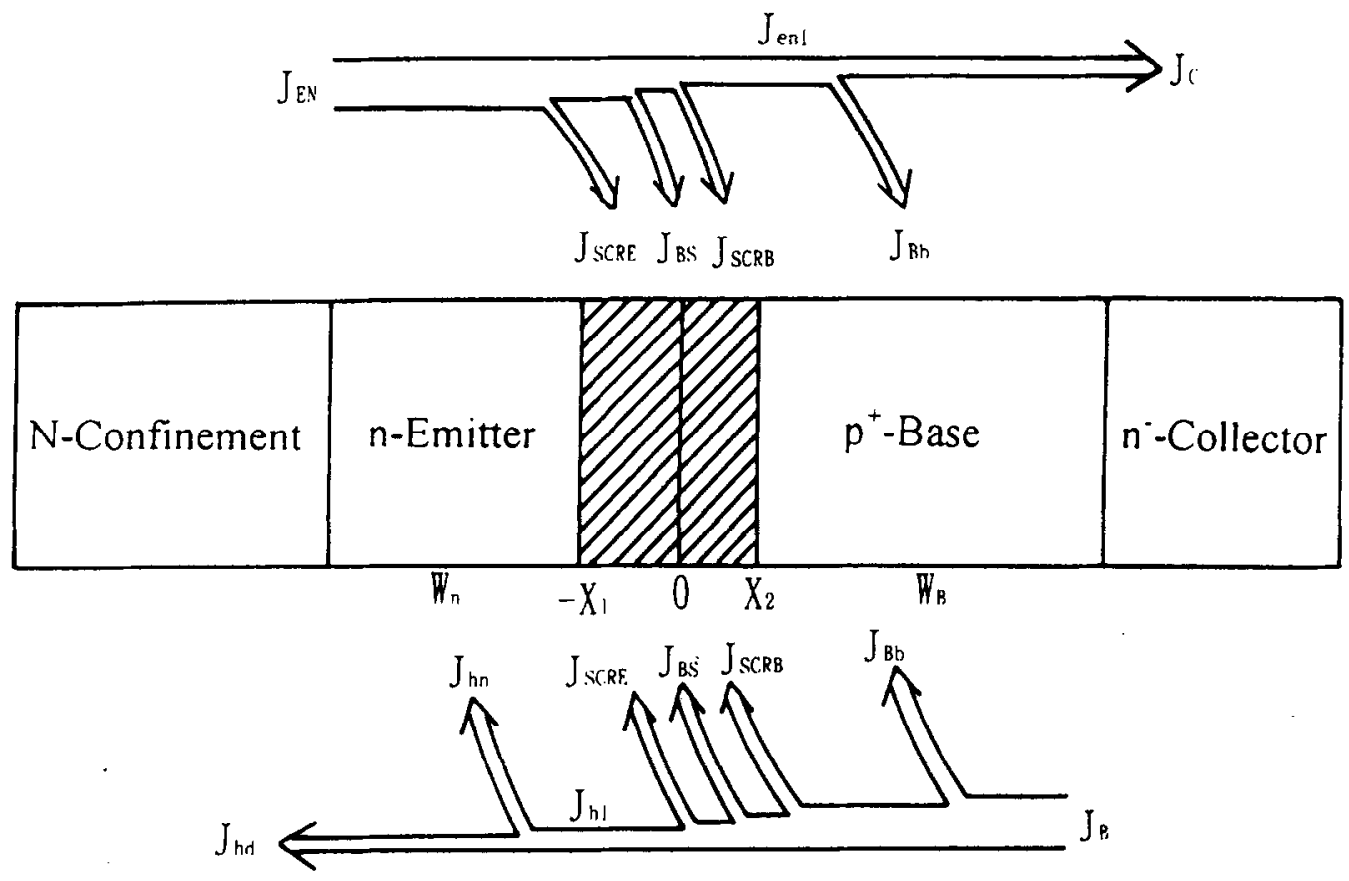

Fig. 2. Schematic illustration of the conduction components of the studied structure.

devices. The calculated conduction band diagrams are illustrated in Fig. 1. The potential spike is not presented under equilibrium of these devices. However, a potential spike may exist for the AlInAs/GaInAs HEBT under large $V_{\mathrm{BE}}$ voltage owing to the large $\Delta E_{\mathrm{c}}$ value. This decreases the emitter injection efficiency. In addition, an n-emitter layer inserted between confinement and base layer would increase charge storage effect and recombination current especially for the short hole diffusion length in the AlInAs/GaInAs HEBT

Figure 2 illustrates the conduction current components of the studied structure. The holes current consists of the thermal-diffusion current $\left(J_{h d}\right)$, the neutral-emitter recombination current $\left(J_{\mathrm{hn}}\right)$, the space-charge region recombination current $\left(J_{\mathrm{SCR}}\right)$, the base surface recombination current $\left(J_{\mathrm{BS}}\right)$ and the recombination current in the bulk quasi-neutral base $\left(J_{\mathrm{Bb}}\right)$. These components of base current can be expressed as follows.

The hole thermal-diffusion current $J_{\text {hd }}$ is dominant:

$$
J_{\mathrm{hd}}=J_{\mathrm{hi}}\left(1-\frac{W_{n}^{2}}{2 L_{\mathrm{p}}^{2}}\right) \exp \left(-\frac{\Delta E v}{\mathrm{~K} T}\right)
$$

with

$$
J_{\mathrm{h} 1}=\frac{q D_{\mathrm{p}} n_{\mathrm{ic}}^{2}}{Q_{\mathrm{E}}} \exp \left(\frac{-q V_{1}}{\mathrm{~K} T}\right)\left[\exp \left(\frac{q V_{\mathrm{BE}}}{\mathrm{K} T}\right)-1\right]
$$

where $D_{\mathrm{p}}, Q_{\mathrm{E}}$ and $n_{\mathrm{ie}}$ are the hole diffusion coefficient, emitter Gummel number and emitter effective intrinsic concentration, respectively. $V_{1}$ is the portion SSE 39:12-B of external voltage $V_{\mathrm{BE}}$ across the heterojunction only. The neutral-emitter recombination current $J_{\mathrm{hn}}$ is

$$
J_{\mathrm{hn}}=J_{\mathrm{hi}}\left(\frac{W_{\mathrm{n}}^{2}}{2 L_{\mathrm{p}}^{2}}\right)
$$

where $W_{\mathrm{n}}$ is the neutral-emitter thickness and $L_{\mathrm{p}}$ is the hole diffusion length in neutral-emitter region.

The space-charge region recombination current $J_{\text {SCR }}$ can be approximated as

$$
J_{\mathrm{SCR}}=q \int_{-X 1}^{0} U_{\mathrm{SCRE}} \mathrm{d} x+q \int_{0}^{X 2} U_{\mathrm{SERB}} \mathrm{d} x
$$

with

$$
U_{\mathrm{SERE}}=0.5 \sigma_{\mathrm{n}} V_{\mathrm{n}} N_{\mathrm{t}} n_{\mathrm{ic}} \exp \left(\frac{V_{\mathrm{BE}}}{2 V_{\mathrm{T}}}\right)
$$

and

$$
U_{\mathrm{SERB}}=0.5 \sigma_{\mathrm{n}} V_{\mathrm{n}} N_{\mathrm{t}} n_{\mathrm{ib}} \exp \left(\frac{V_{\mathrm{BE}}}{2 V_{\mathrm{T}}}\right)
$$

where $X 1$ and $X 2$ are the depletion thickness at emitter and base region, respectively. $\sigma_{\mathrm{n}}, N_{\mathrm{t}}$ and $n_{\mathrm{bb}}$ are the capture cross section, trapping density and base effective intrinsic concentration, respectively. The first and second terms at the right-hand-side of eqn (5) are the space-charge recombination current at the emitter and base region, respectively. 
The base surface recombination current $J_{\mathrm{BS}}$ is given as

$$
J_{\mathrm{BS}}=\frac{I^{*}}{A} \exp \left(\frac{V_{\mathrm{BE}}}{V_{\mathrm{T}}}\right)
$$

where $I^{*}$ is an empirical parameter and $A$ is the emitter junction area. The recombination current in the bulk quasi-neutral base $J_{\mathrm{Bb}}$ can be determined

$$
J_{\mathrm{Bb}}=J_{\mathrm{en}} \frac{W_{\mathrm{B}}^{2}}{2 L e^{2}}
$$

where $J_{\mathrm{en} 1}$ is the electron current at the base depletion edge. $L e$ is the electron diffusion length. Combining the eqns (2)-(9), the total base current $J_{B}$ is

$$
J_{\mathrm{B}}=J_{\mathrm{nd}}+J_{\mathrm{hn}}+J_{\mathrm{scr}}+J_{\mathrm{Bs}}+J_{\mathrm{Bb}} .
$$

In contrast, the electron current at base depletion edge can be expressed as

$$
J_{\mathrm{en} 1}=\frac{q D_{\mathrm{e}} n_{\mathrm{b}}^{2}}{p L e}\left[\exp \left(\frac{q\left(V_{\mathrm{BE}}-V_{1}\right)}{\mathrm{K} T}\right)\right] \operatorname{coth} \frac{W_{\mathrm{B}}}{L_{\mathrm{e}}}
$$

where $D_{\mathrm{e}}, L_{\mathrm{c}}$ and $n_{\mathrm{b}}$ are the electron diffusion coefficient, emitter diffusion length and the base effective intrinsic concentration, respectively. The electron current at collector region $J_{\mathrm{C}}$ is:

$$
J_{\mathrm{C}}=J_{\mathrm{en} 1}-J_{\mathrm{Bb}}
$$

where

$$
J_{\mathrm{en} 1}=J_{\mathrm{EN}}-J_{\mathrm{SCR}}-J_{\mathrm{BS}} \text {. }
$$

Then, the emitter and collector current can be expressed as

$$
I_{\mathrm{E}}=A_{\mathrm{E}}\left(J_{\mathrm{EN}}+J_{\mathrm{ha}}+J_{\mathrm{hn}}\right)
$$

and

$$
\begin{gathered}
I_{\mathrm{C}}=A_{\mathrm{E}} J_{\mathrm{C}} \\
I_{\mathrm{B}}=I_{\mathrm{E}}-I_{\mathrm{C}} .
\end{gathered}
$$

Consequently, the emitter electron injection efficiency $\gamma$ and the current gain $\beta$ can be defined as

$$
y=\frac{A_{\mathrm{E}} J_{\mathrm{en} 1}}{I_{\mathrm{E}}}
$$

and

$$
\beta=\frac{I_{\mathrm{C}}}{I_{\mathrm{B}}}
$$

Figures 3(a) and (b) show the calculated $I_{C}, I_{B}$, and $\gamma$ vs $V_{\mathrm{BE}}$ for the AlGaAs/GaAs and AlInAs/GaInAs HEBTs, respectively. The current gain could increase with increasing $V_{\mathrm{BE}}$ and the maximum value is about 360 for the studied AlGaAs/GaAs HEBT. On the contrary, the current gain could decrease with increasing the $V_{\mathrm{BE}}$ voltage for the studied AllnAs/ GaInAs HEBT. Obviously, the $\gamma$ value slightly decreases with increasing the $V_{\mathrm{BE}}$ voltage for the AlInAs/GaInAs HEBT. However, the $\gamma$ value of the
AlGaAs/GaAs HEBT increase rapidly initially and approaches unity when the $V_{\mathrm{BE}}$ voltage is larger than $0.7 \mathrm{~V}$. Figures $4(\mathrm{a})$ and (b) illustrate the ratio of the base component current to the total base current of the studied devices. Clearly, for the AlInAs/GaInAs HEBT the neutral-emitter recombination current is a significant factor for dominating the total base current. It is attributed to the shorter hole diffusion length of InGaAs. That is to say, during the holes injected from the base region into the confinement layer, most of holes recombined with electrons in the neutral-emitter region. Especially, the recombination current increases under larger $V_{\mathrm{BE}}$ voltage bias. Because the neutral-emitter region could be extended at larger $V_{\mathrm{BE}}$, the neutral-emitter recombination current is enhanced and the current gain as well as the $\gamma$ value could be degraded with increasing $V_{\mathrm{BE}}$ voltage. On the other hand, the current gain increases with small $V_{\mathrm{BE}}$ and reaches to a fixed value at $V_{\mathrm{BE}}=0.8 \mathrm{~V}$ for the AlGaAs/GaAs HEBT. The main component of $I_{\mathrm{B}}$ is the base surface recombination current. That is because the hole diffusion length is larger than the emitter thickness which leads to less recombination at the neutral-emitter region.

\section{EXPERIMENTAL RESULTS AND DISCUSSION}

The common-emitter current-voltage (I-V) characteristics of the AlGaAs/GaAs and AllnAs/GaInAs HEBTs, measured by a Tektronix 577 curve tracer, are shown in Figs 5(a) and (b), respectively. The maximum common-emitter current gains are of 180 and 25 for AlGaAs/GaAs and AlInAs/GaInAs HEBTs, respectively. It is worth noting that the current gain of AlInAs/GaInAs HEBT decreases with increasing the base current $I_{B}$. From the above analysis, there are a number of holes recombined in the neutral-emitter region. This certainly degrades the current gain. The experimental Gummel plot of $I_{C}$ and $I_{\mathrm{B}}$ vs $V_{\mathrm{BE}}$ are depicted in Fig. 6 . For the $\mathrm{AlGaAs} / \mathrm{GaAs}$ HEBT, the ideal factor of $I_{\mathrm{C}}$ is nearly unity, which is attributed to the elimination of potential spike and low recombination currents. The ideal factors of $I_{\mathrm{B}}$ are 1.8 and 1.2 at small $V_{\mathrm{BE}}$ and $V_{\mathrm{BE}}>0.75 \mathrm{~V}$, respectively. That is because the space-charge is important at small $V_{\mathrm{BE}}$ voltage regime and the base surface recombination current dominates the total base current at larger $V_{\mathrm{BE}}$ voltage, as revealed in Fig. 4(a). On the other hand, a non-1KT ideal factor of $I_{\mathrm{C}}$, due to the potential spike and low emitter injection efficiency, of the AlInAs/GaInAs HEBT is found. Moreover, an ideal factor 1.1 of $I_{\mathrm{B}}$ is obtained. So, the neutral-emitter recombination current strongly dominates the total base current of the AlInAs/GaInAs HEBT. Consequently, for the AlInAs/GaInAs HEBT, at large $V_{\mathrm{BE}}$ voltage the emitter thickness of $500 \AA$ will degrade the emitter injection efficiently. In contrast, the $\mathrm{AlGaAs} / \mathrm{GaAs}$ HEBT behaves as a regular transistor due to the low 


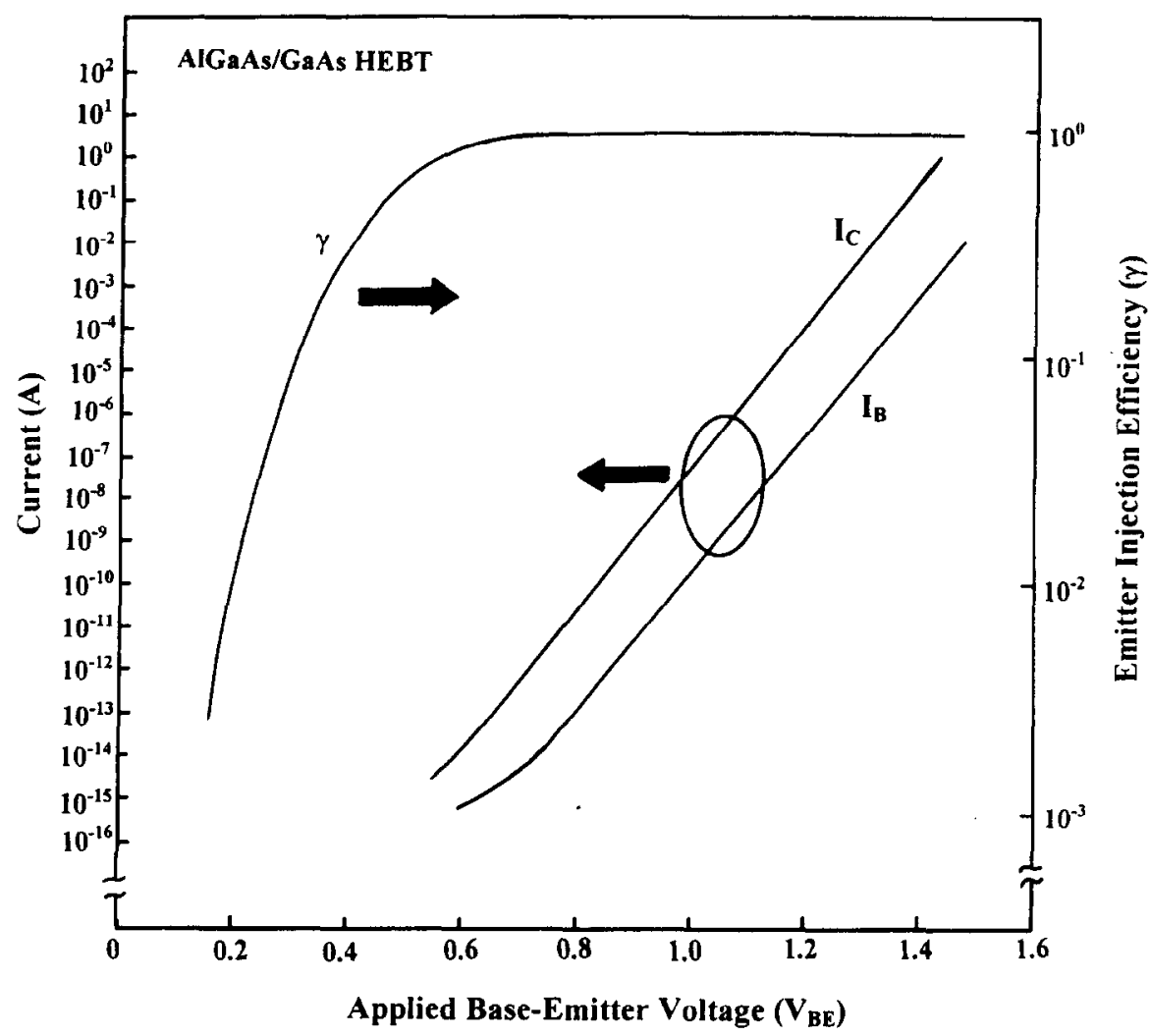

(a)

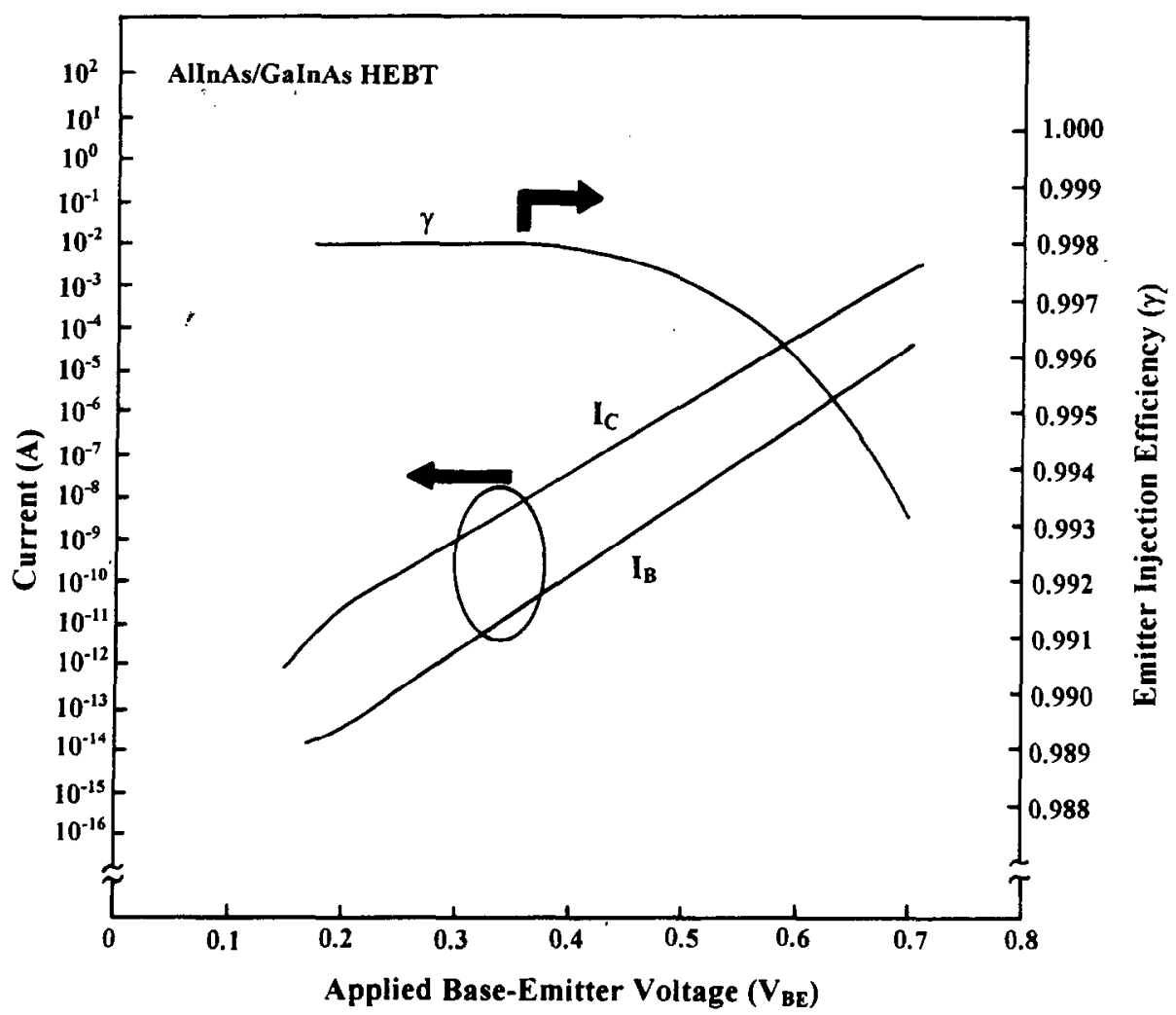

(b)

Fig. 3. The calculated $I_{\mathrm{C}}, I_{\mathrm{B}}$ and $\gamma$ vs $V_{\mathrm{BE}}$ voltage for: (a) AlGaAs/GaAs; and (b) AlInAs/GaInAs HEBT. 


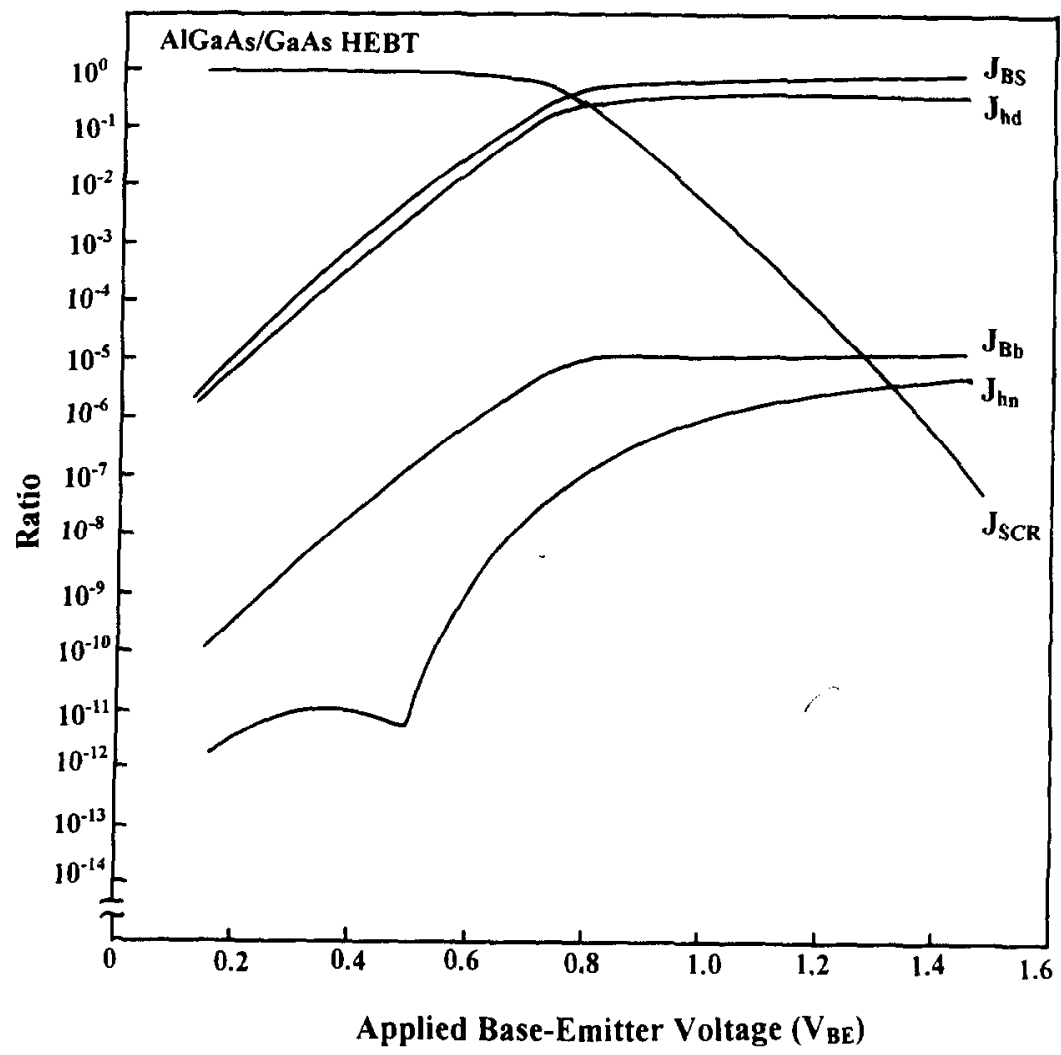

(a)

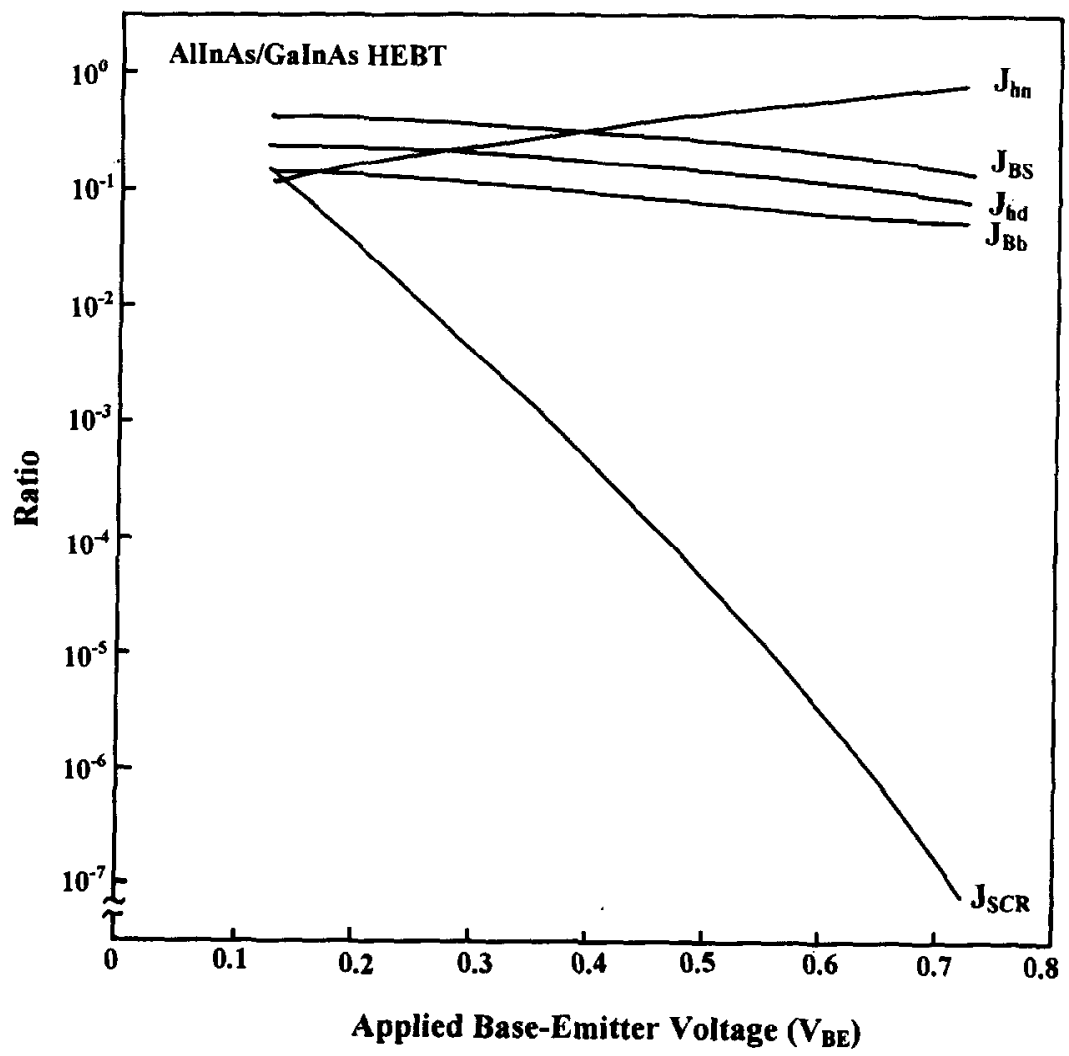

(b)

Fig. 4. The ratio of the base component current to the total base current for: (a) AlGaAs/GaAs; and (b) AllnAs/GaInAs HEBTs. 
recombination current in the neutral-emitter region. The offset voltage $\Delta V_{C E}$ of $40 \mathrm{mV}$ is obtained for the studied AlInAs/GaInAs HEBT which is smaller than that of AlGaAs/GaAs HEBT $(80 \mathrm{mV})$. Because the surface recombination current of AlInAs/InAs junction is so small that it can be neglected and the potential spike is eliminated at small $V_{\mathrm{BE}}$ voltage, the offset voltage $\Delta V_{\mathrm{CE}}$ can be reduced. On the contrary, the surface recombination current of $\mathrm{AlGaAs} / \mathrm{GaAs}$ region is obvious which gives a relatively higher offset voltage of the AlGaAs/GaAs HEBT. From the viewpoint of the transistor performances, the emitter thickness of $500 \AA$ is appropriate for the AlGaAs/GaAs HEBT. Moreover, for the AllnAs/GaInAs HEBT the value could be reduced to $400 \AA$ or less length to achieve higher current gain. It is known that, from the description as mentioned above, the experimental results are consistent with the theoretical analysis.

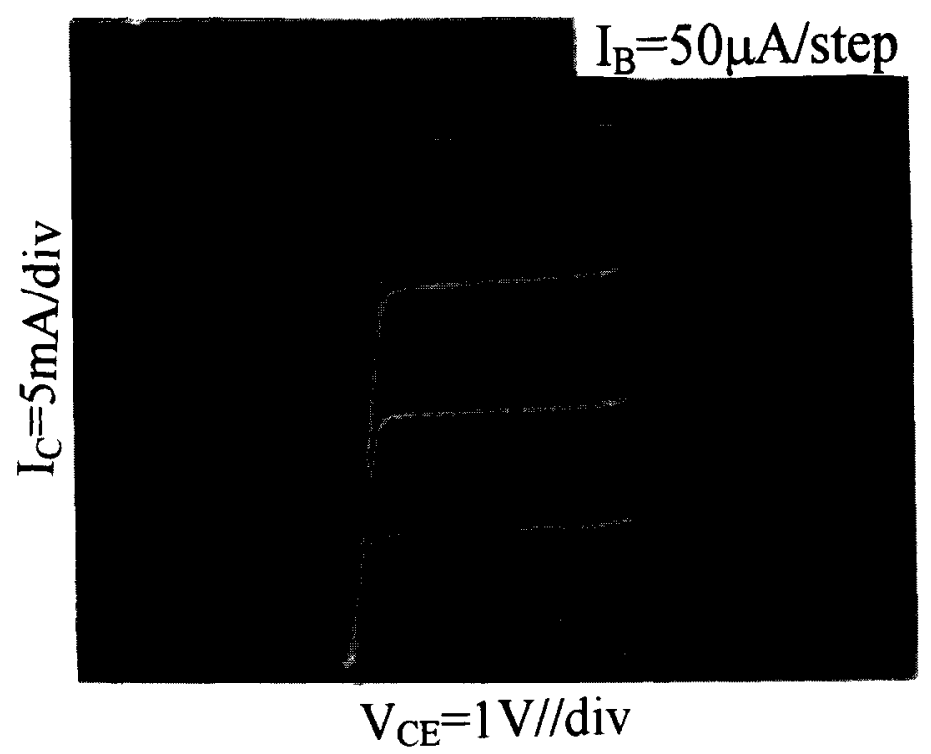

(a)

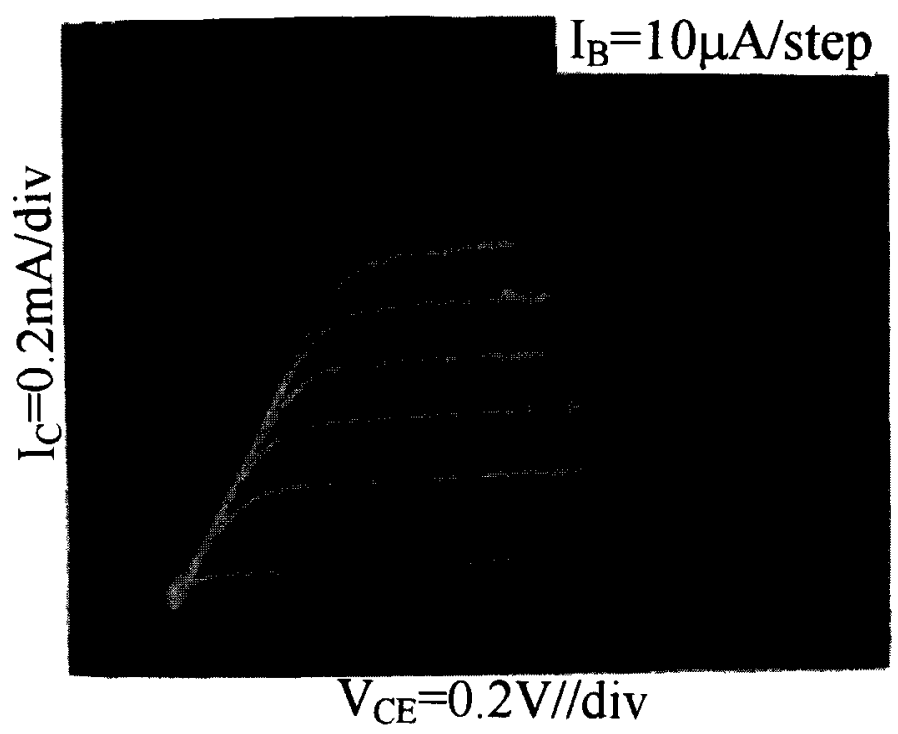

(b)

Fig. 5. Experimental common-emitter current-voltage (I-V) characteristics for: (a) the AlGaAs/GaAs; and (b) AlInAs/GaInAs HEBT. 


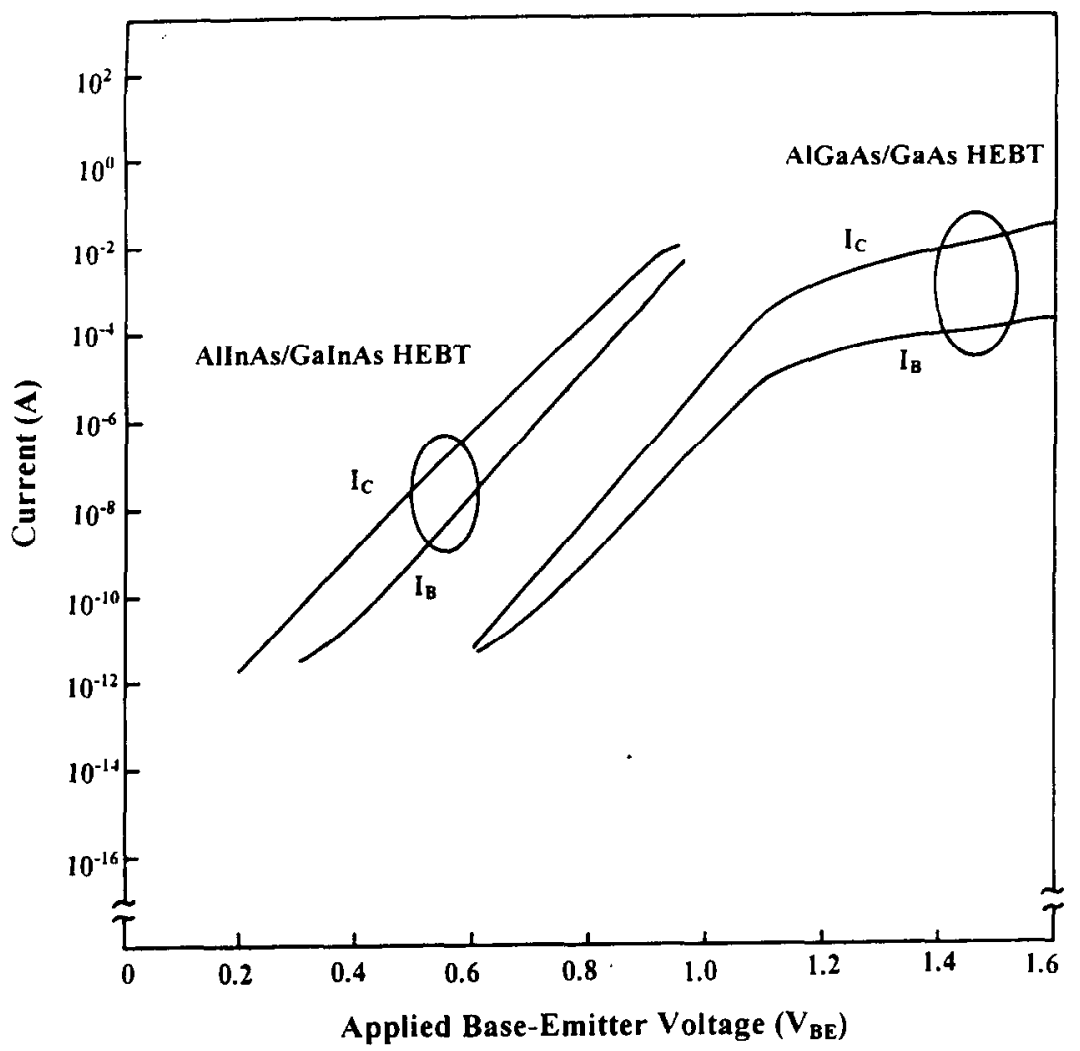

Fig. 6. The experimental Gummel plot of $I_{\mathrm{C}}$ and $I_{\mathrm{B}} \mathrm{vs} V_{\mathrm{BE}}$ voltage.

\section{CONCLUSIONS}

The theoretical analysis and experimental results are developed to describe the recombination current characteristics of the studied devices. For the AlGaAs/GaAs HEBT, the hole diffusion length is larger than the emitter thickness. Thus, the neutral-emitter recombination current is low and the high emitter injection efficiency and current gain are achieved. Experimentally, our AlGaAs/GaAs HEBT shows a high current gain of 180 . On the contrary, for the AlInGa/GaInAs HEBT, the neutral-emitter recombination current dominates the base current at large $V_{\mathrm{BE}}$ bias. So, the emitter injection efficiency is decreased and the transistor characteristics are degraded. Hence, the emitter layer thickness must be carefully designed to achieve excellent transistor behaviors. The experimental results are consistent with theoretical analysis. Based on the accurate analysis, an appropriate HEBT design could improve the transistor properties and provides a promise for the digital and analogue circuit applications.
Acknowledgement-This study was supported by the National Science Council of the Republic of China under contract no. NSC 85-2215-E-006-023.

\section{REFERENCES}

1. S. S. Tan and A. G. Milnes, IEEE Trans. Electron. Device ED-3, 1289 (1983).

2. P. M. Asbeck, M. F. Chang, K. C. Wang, D. L. Miller, G. J. Sullivan, N. H. Sheng, E. Sovero and J. A. Higgins, IEEE Trans. Electron. Device ED-34, 2571 (1987).

3. C. Takano, K. Taira and H. Kawai, IEEE Electron. Device Lett. EDL-9, 142 (1988).

4. L. F. Luo, H. L. Evans and E. S. Yang, IEEE Trans. Electron. Device ED-36, 1844 (1989).

5. W. C. Liu and W. S. Lour, Solid State Electron. 7, 717 (1991).

6. H. R. Chen, C. Y. Chang, C. P. Lee, C. H. Hung, T. S. Tang and K. L. Tsai, IEEE Electron. Device Lett. 15, 336 (1994).

7. Y. F. Yang, C. C. Hsu and E. S. Yang, IEEE Trans. Electron. Device ED-41, 643 (1994).

8. M. E. Hafizi, C. R. Crowell and M. E. Grupen, IEEE Trans. Electron. Device ED-37, 2121 (1990).

9. B. R. Ryum and I. M. Abdel-Motaleb, IEE Proceedings-G 115 (1991).

10. T. W. Lee and P. A. Houston, IEEE Trans. Electron. Device ED-40, 1390 (1993). 